Musées, Patrimoine et Culture scientifiques et techniques

$154 \mid 2014$

juillet-août 2014

\title{
Les peaux humaines tatouées, un patrimoine caché
}

par Éloïse Quétel

\section{OpenEdition \\ Journals}

Édition électronique

URL : http://journals.openedition.org/ocim/1431

DOI : 10.4000/ocim. 1431

ISSN : 2108-646X

Éditeur

OCIM

Édition imprimée

Date de publication : 25 juillet 2014

ISSN : 0994-1908

Référence électronique

par Éloïse Quétel, «Les peaux humaines tatouées, un patrimoine caché », La Lettre de l'OCIM [En ligne], 154 | 2014, mis en ligne le 25 juillet 2016, consulté le 19 avril 2019. URL : http:// journals.openedition.org/ocim/1431; DOI : 10.4000/ocim.1431

Ce document a été généré automatiquement le 19 avril 2019

Tous droits réservés 


\title{
Les peaux humaines tatouées, un patrimoine caché
}

\author{
par Éloïse Quétel
}

Portrait of James Cook (1728-1779) par Nathaniel Dance-Holland, National Maritime Museum, United Kingdom.

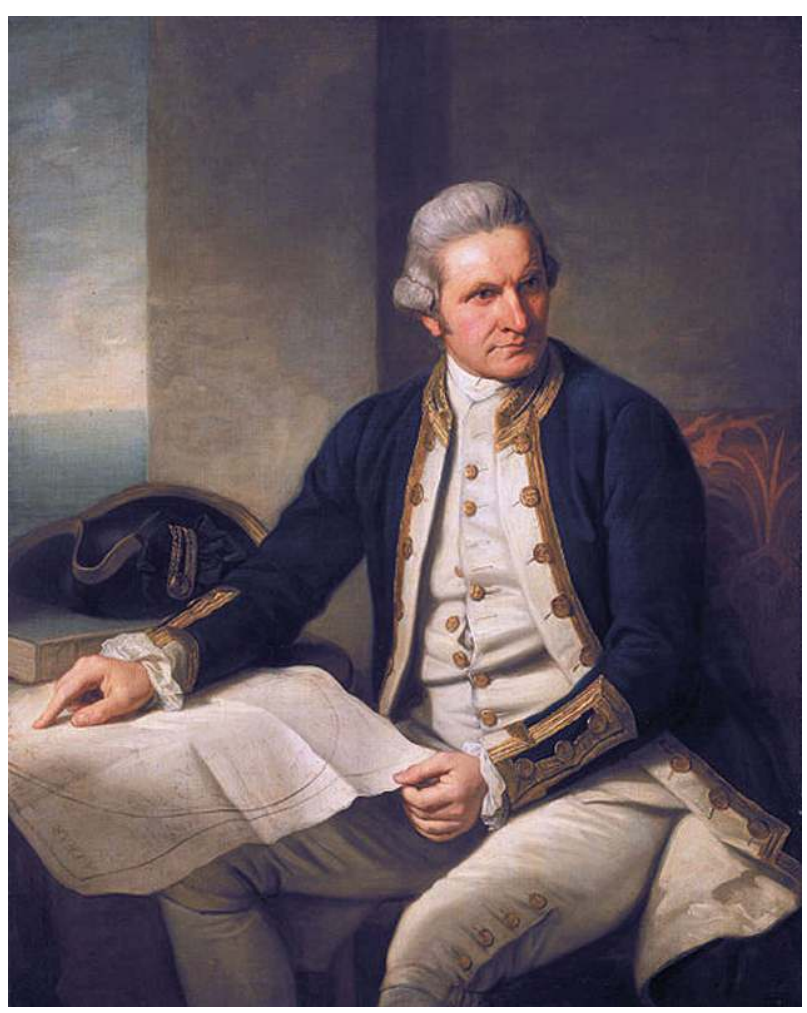

(c) DR

1 Il existe de nombreuses variétés de collections de restes humains: des collections ostéologiques, des préparations anatomiques et anthropologiques, des momies, des 
artefacts culturels et cultuels, des préparations en fluide, des objets de curiosité... Ces collections sont présentes dans de nombreux musées en France et dans le monde. Nous savons par ailleurs qu'au sein de ces collections, il existe des spécimens cachés, issus d'un patrimoine que l'on dit « noir », les peaux humaines tatouées en font partie.

\section{Un historique singulier}

2 Le marquage corporel est une tradition qui existe depuis toujours et dans toutes les cultures, que ce soit la peinture sur le corps, les scarifications, les brûlures, le bronzage ou le tatouage. La volonté de modifier l'enveloppe corporelle, de manière culturelle, cultuelle, collective ou individuelle a perduré jusqu'à nos jours.

Le tatouage est une pratique qui a été popularisée par James Cook, un explorateur qui a réalisé de nombreux voyages dans les îles du Pacifique au XVIII ${ }^{\mathrm{e}}$ siècle. Aux yeux des Occidentaux, ce marquage est l'apanage du primitif et suscite une grande curiosité, à la fois sur la question du phénomène vivant (ce que l'on appellera par la suite les «zoos humains ») mais aussi sur la représentation des tatouages. C'est cette fascination qui va conduire les explorateurs, marins du " petit peuple» mais aussi aristocrates, à se faire tatouer lors de leur s expéditions sur les îles. C'est lors de leur retour en Occident que l'impact de leurs tatouages va déclencher une vague de mimétisme dans différentes sphères de la population, au mépris de ce que cela pouvait encore signifier dans les mœurs des autochtones.

4 Le tatouage en Occident va demeurer une marque d'infamie et d'opprobre pendant de nombreuses décennies encore. Même si certains membres de l'aristocratie se distinguent par leurs tatouages, la recrudescence du phénomène va surtout être observée auprès du " petit peuple». Ce sont principalement les marins, compagnons, militaires, prisonniers, prostituées et artisans qui vont marquer à même leurs peaux les différentes étapes et événements de leur vie. 
Cesare Lombroso, 1835-1909 (extrait du site: Images from the History of Medicine).

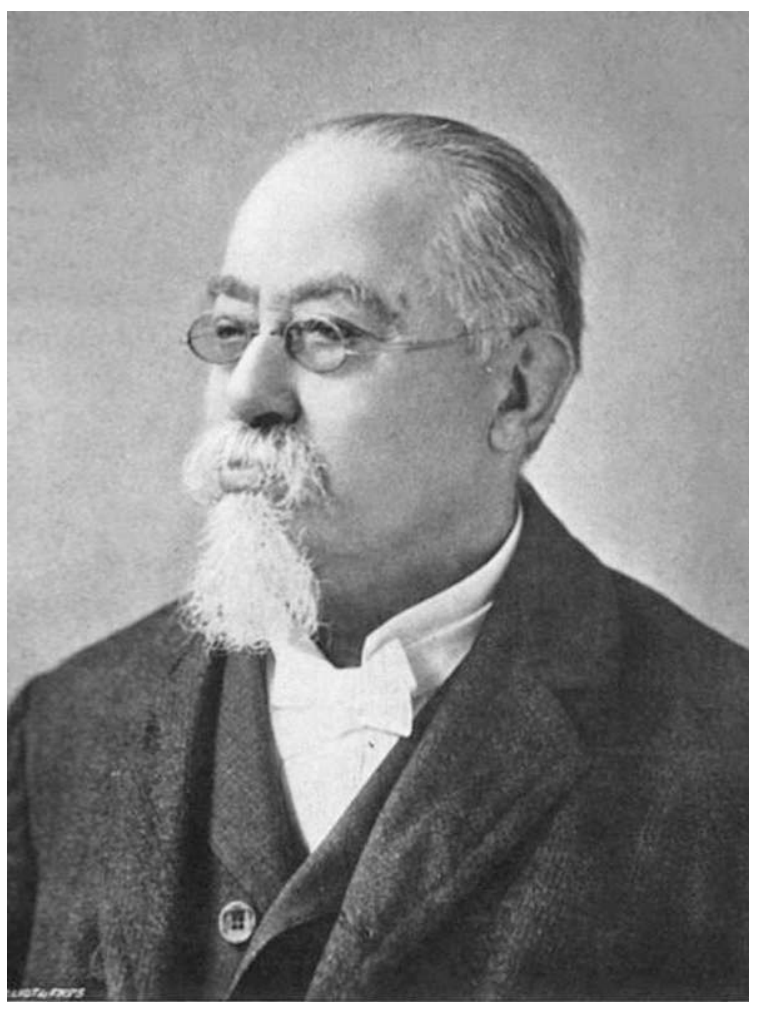

(c) Deschiens

Alexandre Lacassagne, 1843-1924 (extrait du site : Images from the History of Medicine).

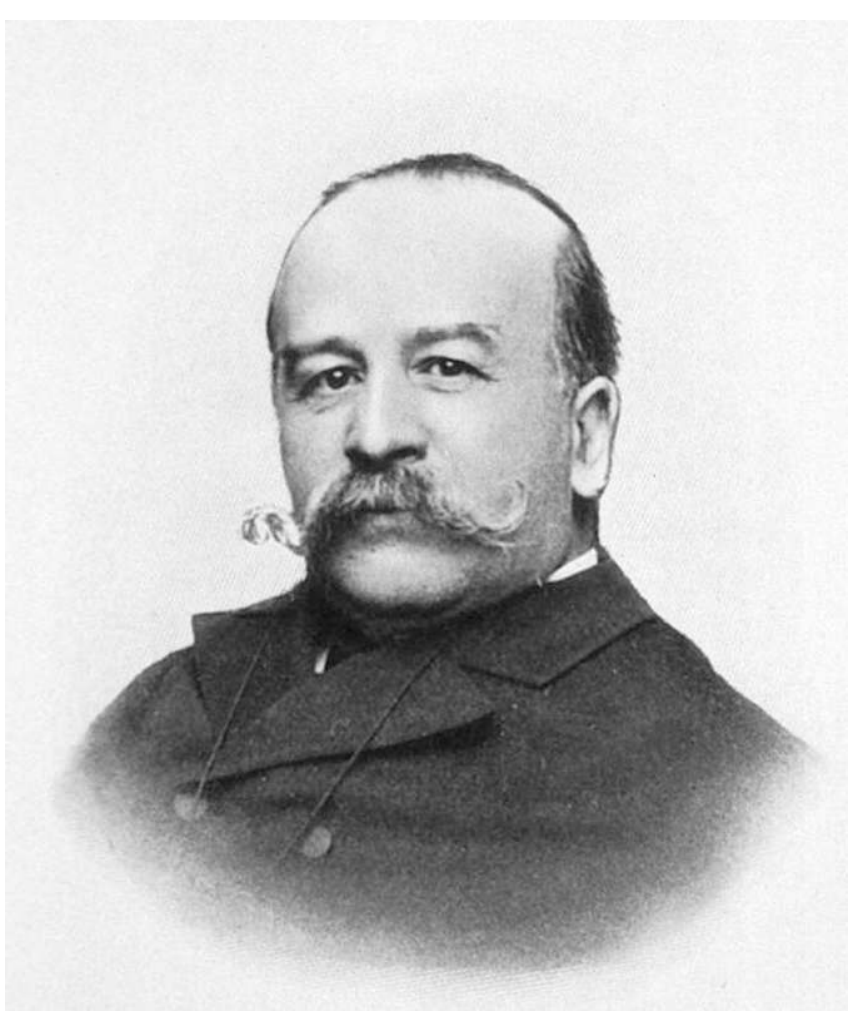

(c) Deschiens 
Glissant jusqu'à la fin du XIX ${ }^{e}$ siècle, cette culture a stigmatisé une catégorie sociale qui s'est vue disséquée par les précurseurs de l'anthropologie criminelle pendant plusieurs décennies. Ce sont Cesare Lombroso et Alexandre Lacassagne, tous deux médecins légistes et criminologues, qui vont établir les premières théories sur le tatoué criminel. À l'aide d'un procédé simple, le décalquage des motifs sur le corps des tatoués, ils ont recensé les formes, les couleurs, et les motifs caractérisant les tatouages. Lacassagne a notamment réuni de cette manière plus de 2000 tatouages sur 150 individus. Cette collection, comme il l'appelait, lui a permis d'établir des conclusions d'une certaine importance du point de vue médico-judiciaire. "Ce que prouve habituellement la présence des tatouages c'est que l'individu qui en est porteur appartient à un milieu de faible instruction et de médiocre éducation. Et il ne fait pas oublier que c'est dans ce même milieu que se recrute la majorité des criminels ${ }^{1}$.

6 La seconde méthode d'étude, fut celle du prélèvement après la mort ; les peaux étaient ôtées du corps des tatoués afin d'être observées et quelques fois disséquées. Les peaux, une fois prélevées et préparées (parfois en fluide, et le plus souvent une solution formolée, ou sèches après un tannage et une tension sur cadre) étaient conservées et ont peu à peu constitué des collections singulières. Prélever la peau du tatoué, signifiait conserver le support d'origine du tatouage. En effet, on constate que même si l'image photographique ou le décalquage sont de bons outils didactiques, ils ne peuvent pas remplacer un spécimen authentique qui représente la diversité naturelle qui nous entoure.

\section{Un cas d'étude}

7 Afin d'étayer nos propos, nous avons travaillé sur cinq peaux humaines tatouées conservées au sein des collections du musée d'anatomie Testut-Latarjet de Lyon. Il s'agit d'une collection universitaire issue des spécimens collectés par la société de médecine et considérablement enrichie au XIX ${ }^{e}$ siècle par Léo Testut et André Latarjet (dont le musée conservera les noms). Cette collection a notamment bénéficié de l'ajout des spécimens collectés par Alexandre Lacassagne dans son musée de médecine légale. Lorsque ce musée fut fermé et que la plupart des pièces qui le composaient furent dispersées ou tout simplement détruites, les quelques peaux tatouées subsitant encore furent récupérées et intégrées à la collection anthropologique du musée d'Anatomie Testut-Latarjet.

Dans les vitrines de la section d'anthropologie criminelle sont conservés des rapports de police avec les couteaux "incriminés", des crânes perforés par des balles, des crânes reconstitués après morcellement, des plâtres représentant les médecins illustres qui ont enseigné à Lyon, des échantillons de cheveux, et des balles. On observe aussi différents types de peaux, scalps, têtes momifiées ainsi que des peaux humaines tatouées.

9 La collection comporte dix-sept peaux humaines tatouées sèches, dont quatre grandes peaux et quarante trois échantillons conservés en fluide, issus de recherches dermatologiques. Hormis la provenance de certaines peaux issues de la collection Lacassagne, aucune autre information n'existe: on ne sait ni l'origine, ni la date du prélèvement des peaux. 
Vues recto et verso des cinq peaux.

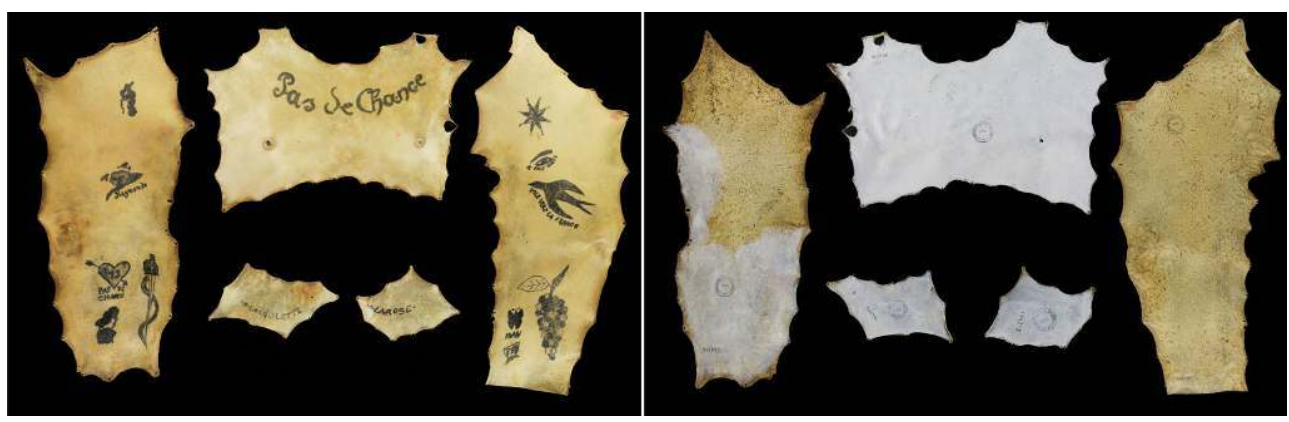

(c) Éloïse Quétel nous ont amené à réaliser un recensement des différentes collections existantes dans le monde (voir encadré). Grâce à ce panel d'exemples, nous avons déterminé que la période de prélèvement était située entre 1850 et 1950 (en prenant en compte notamment les peaux prélevées lors de la Seconde Guerre mondiale, sous l'ordre de Ilse Koch, femme du commandant Nazi Karl-Otto Koch à Buchenwald), et plus particulièrement entre 1890 et 1920 pour la plupart des collections.

Les cinq échantillons de peau humaine tatouée que nous avons sélectionné pour réaliser notre étude ont été prélevés sur le même individu. À l'aide d'un scalpel, la peau du torse, des deux bras, et des deux dessus de pieds a été ôtée, préparée et conservée de manière sèche.

d'en apprendre un peu plus sur la collection du musée d'anatomie de Lyon, nous avons questionné le conservateur, Jean-Christophe Neidhardt et avons découvert que ces cinq peaux n'étaient pas issues de la collection Lacassagne, mais d'un prélèvement beaucoup plus récent, réalisé à la fin des années 1990 .

Les peaux humaines conservées dans le monde

Pour en faire très rapidement le tour, hormis la collection de Lyon, il y a tout d'abord le Science Museum qui conserve plus de 300 fragments secs, le département de pathologie de l'université de Médecine de Tokyo qui possède 120 pièces sèches (dont plusieurs sont de grandes pièces, dos complets, jambes et bras du même individu), et la série de quatre vingt trois peaux sèches de la collection des arts bruts de Lausanne. Par ailleurs, le Medizinhistorisches Museum de Berlin préserve des spécimens en fluide, tout comme le Pathologisch-anatomische Bundesmuseum (ou Narrenturm) de Vienne et l'université de Jagiellonian en Pologne. Le musée de l'Homme quant à lui, conserve à peu près 50 pièces sèches, et le conservatoire d'Anatomie de Montpellier (tout comme le muséum d'Histoire Naturelle de Rouen) n'en conserve qu'une seule, sèche. Enfin, le musée d'Anatomie Luigi Rolando de l'université de Turin, le musée du Tatouage d'Amsterdam (aujourd'hui fermé faute de subvention), le musée d'Art et d'Histoire Guy-Baillet à Langres, et le musée national de la Marine à Paris comptent également des spécimens secs et en fluide. 


\section{Une question d'esthétique}

13 Comme la plupart des musées d'anatomie et de médecine, le musée d'anatomie TestutLatarjet est situé sur le même site qu'une faculté de Médecine (ici l'université Claude Bernard). Le personnel du musée est ainsi régulièrement en contact avec les étudiants, médecins et chercheurs travaillant à la faculté, mais aussi avec les techniciens du laboratoire d'anatomie jouxtant les locaux du musée. Ainsi, lorsqu'à la fin des années 1990, la réfection des locaux du laboratoire d'anatomie fut lancée, et que les congélateurs furent vidés afin d'être renouvelés, plusieurs corps et fragments de corps furent envoyés au crématorium afin d'être inhumés. Ce ne fut pas le cas d'un corps en particulier dont la peau fut conservée. Sorti du congélateur au moment où Jean-Christophe Neidhardt, le conservateur, était de passage, ce corps tatoué l'a intrigué. La facture des dessins était analogue à celle des peaux conservées par Alexandre Lacassagne un siècle plus tôt. Guidé par la volonté d'agrémenter la collection de nouveaux spécimens, le conservateur, accompagné de deux étudiants en médecine préleva ces 5 morceaux de peaux.

Installés au laboratoire d'anatomie afin d'effectuer les prélèvements, le conservateur et les étudiants ont procédé en plusieurs étapes: la première fut celle de la dénudation durant laquelle les différents fragments de peaux furent prélevés avec un scalpel, des pinces et des ciseaux. Ils ont ensuite passé les peaux sous l'eau afin de les rincer, et, avec un scalpel, des pinces et des ciseaux, ont enlevé l'hypoderme restant. Enfin, dans le but de les fixer (couper le processus de putréfaction), les peaux ont été plongées plusieurs jours dans une solution à base de formaldéhyde et d'eau. Une fois fixées, les peaux ont été clouées et tendues sur des planches en bois afin de les faire sécher et leur redonner leur taille initiale.

On constate, par le truchement de la découpe, que le conservateur a délibérément souhaité conserver la forme de l'emplacement d'origine où les peaux ont été ôtées. Ainsi, la peau du torse nous suggère la forme d'un "marcel ", celle des bras est complète, découpée du dessous de l'aisselle au poignet, seule la peau des dessus des pieds présente une forme abstraite, ne figurant que de simples « rectangles ».

\section{Une iconographie}

16 "Dans ces dessins emblématiques, il y a, ainsi que nous l'avons fait voir, une source précieuse de renseignements sur la nature des idées morales des tatoués: leur pensée ordinaire, les images qui leur sont chères, leurs souvenirs intimes, parfois inavouables, et même leurs projets de vengeance cyniquement formulés. [...] Le médecin légiste doit savoir tirer parti de tous les détails. [...] Les tatouages, [...] par leur variété et leur nombre, marquent souvent les étapes de la vie d'un individu, et parfois sa nature morale. Ce sont des cicatrices parlantes. En médecine judiciaire, il n'existe pas de meilleurs signes d'identité par leur caractère de permanence, de durée, la difficulté à les faire disparaître $»^{2}$.

17 Comme nous l'avons vu, les tatouages ont été répertoriés et étudiés à la fin du XIX et au début du $\mathrm{XX}^{\mathrm{e}}$ siècle afin de catégoriser et classer le passé des tatoués. Lorsque nous avons étudié les motifs «encrés» dans ces 5 peaux, nous avons tenté de saisir une brève biographie de notre individu tatoué. Ils se déclinent en diverses catégories : 
- les inscriptions : «Pas de Chance » sur le torse, « À la violette », « À la rose » sur les pieds, et «M.A.V» (Mort Aux Vaches), sur le bras gauche;

- les inscriptions mêlées aux emblèmes avec l'œil souligné d'un «Je fais gaffe » et l'hirondelle avec l'épitaphe «Vole vers la France» sur le bras gauche, la pensée entourée d'un «À ma Raymonde » et un cœur percé d'une flèche à l'intérieur duquel le nombre 13 est inscrit sur le bras droit ;

- les emblèmes seuls : la rose des vents, le papillon, la grappe de raisin ainsi que le serpent enroulé autour d'une épée sur le bras gauche ;

- les représentations humaines, avec un buste d'homme fumant une cigarette et deux bustes de femmes dont un portant un chapeau sur le bras droit.

Ces tatouages nous permettent d'imaginer le parcours de cet individu, et le regroupement de certains motifs donne à penser que le tatoué a voulu raconter une histoire bien particulière à même sa peau. Le bras gauche regroupe les tatouages qui parlent d'anarchie face au pouvoir en place (les matons, les policiers), de la colonie pénitentiaire, du voyage à l'étranger, de la vie dans le milieu. Le bras droit parle d'amour, de vengeance et de regret, et le torse porte un tatouage de revendication, un ressentiment porté en toutes lettres près du cœur. Quant aux tatouages sur les pieds, ils montrent l'humour du tatoué. Même si nous n'en sommes pas sûrs, cet homme n'a probablement jamais imaginé que sa peau allait lui survivre et raconter encore et toujours son histoire personnelle...

Conditionnement, détail de la planche qui maintient la peau du bras gauche.

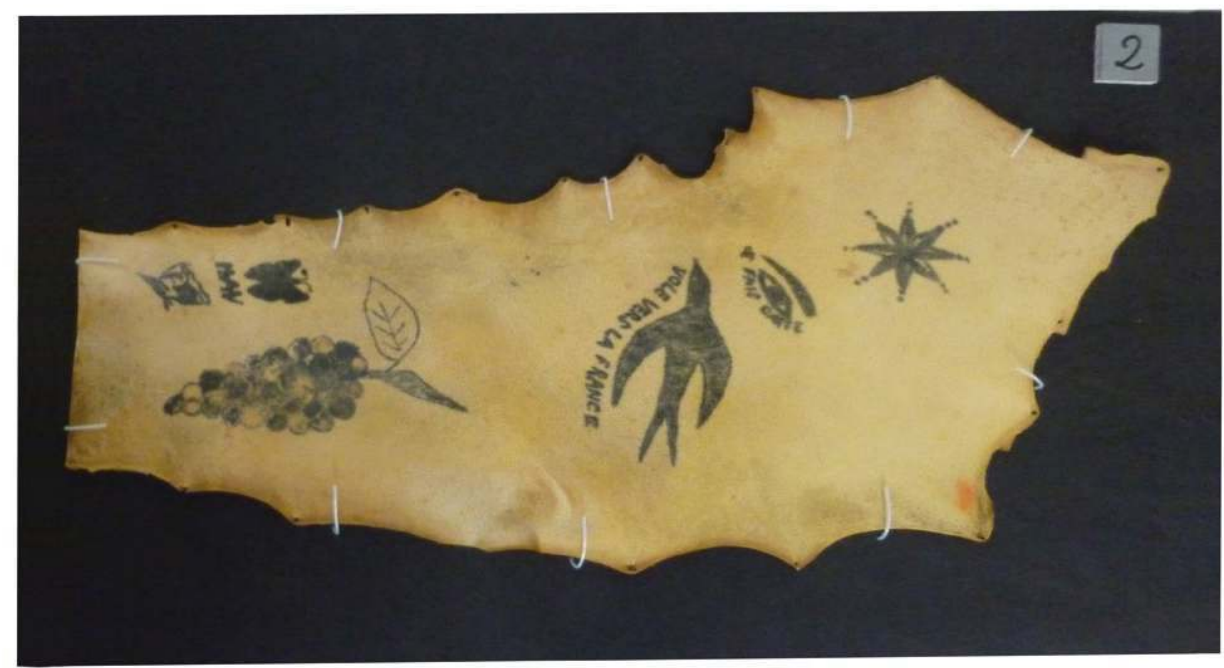

(c) Éloïse Quétel

\section{La conservation}

Une fois prélevées, les peaux ont été préparées et tendues pour être séchées. Elles ont ensuite été badigeonnées d'huile d'olive à la face dans le but de faire ressortir les 
pigments noirs de l'encre, et de cire blanche à chaussure au revers où elles sont les plus fines, afin de les opacifier.

Afin de les intégrer au sein de la collection, chaque fragment de peau a été marqué d'un numéro d'inventaire, une première fois en utilisant un stylo qui a en partie creusé la cire blanche et une seconde fois avec deux tampons, un pour le numéro d'inventaire et un second avec le cachet de l'université Claude Bernard. Ils ont par la suite directement rejoint le reste de la collection, qui est conservée dans une vitrine au sein même de l'espace d'exposition.

21 Le constat d'état des peaux a révélé des altérations structurelles et surfaciques qui sont dues à la fois au mode de préparation des peaux et aux conditions et variations atmosphériques observées au sein du musée. Une ondulation généralisée des peaux, le jaunissement dû à l'oxydation du collagène et de l'huile d'olive, des taches rose orangé issues du double marquage réalisé au stylo (qui a fusé au travers de la cire blanche et du derme) ainsi qu'une fissure au niveau de la peau du coude gauche, sont des altérations structurelles. Un empoussièrement généralisé, des zones encrassées, des particules cristallines sur la quasi-totalité du verso des peaux, et des petits agglomérats de graisse sont des altérations surfaciques.

Nous avons proposé et réalisé deux traitements, un curatif et un préventif: un dépoussiérage à l'aide d'une petite brosse souple et d'un aspirateur à filtre HEPA (High Efficiency Particules Arresting) ainsi qu'un conditionnement adapté, fabriqué sur mesure avec des matériaux inertes et chimiquement neutres.

Comme la particularité de cette collection est qu'elle ne sera probablement jamais exposée (comme c'est le cas pour la plupart des collections de peaux humaines tatouées dans le monde), il fallait en priorité réaliser une unité de stockage pour ces 5 fragments de peau qui leur permettrait d'être protégés des éléments extérieurs pouvant à terme provoquer de futures altérations, comme les insectes, la poussière, mais aussi les manipulations hasardeuses ou les trop grandes variations atmosphériques. Cela a aussi permis de créer un espace de présentation adapté et sécurisé, permettant la lisibilité des fragments sans avoir forcément à les sortir de leur conditionnement.

Un conditionnement adapté aux cinq peaux.

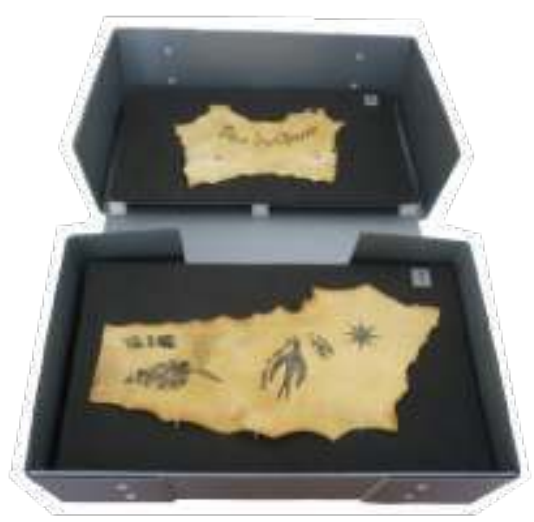

(c) Éloïse Quétel 
24

Ainsi, une boîte en polypropylène cannelé a été conçue avec des planches de consultations individuelles pour chaque fragment ${ }^{3}$. Nous avons utilisé du polypropylène cannelé doublé de mousse de polyéthylène (Plastazote) pour les planches, ainsi que des fils d'acier inoxydable gainé avec du tube de Polytétrafluoroéthylène (PTFE) pour réaliser des appliques réversibles et repositionnables sur le pourtour des éléments afin de les maintenir en place. Les planches de consultation ont été munies au revers de cales en mousse de polyéthyène (Ethafoam ${ }^{\circ}$ ) afin de pouvoir les superposer dans la boîte et les déplacer plus facilement.

Enfin, nous avons décidé de fermer la boîte avec un couvercle rétractable et encastrable sans ajout de matériau tel que le velcro ou les aimants, afin de limiter au maximum les vibrations et les chocs provoqués par ces matériaux à l'ouverture et la fermeture de la boîte. Une signalétique (photographie, descriptif concis des échantillons de peau, sens d'ouverture) a été ajoutée tout autour de la boîte ainsi qu'une fiche récapitulative des préconisations de manipulation à l'intérieur du couvercle, tout ceci dans le but d'une conservation optimale de ces cinq peaux.

\section{L'épineuse question juridique}

Les premières lois françaises qui se sont penchées sur le sujet sont les lois de Bioéthique 16-1 et 16-1-1 du Code civil (introduites respectivement en 1994 et en 2008) qui nous indiquent que le corps humains est indisponible, inviolable et non patrimonialisable. Les règles de l'ICOM $(2.5,3.7$ et 4.3$)$ viennent compléter notre regard sur cette question en énonçant notamment que la conservation et l'exposition des restes humains doivent être réalisées dans les intérêts et croyances de la communauté ou des groupes ethniques et religieux d'origine, avec le plus grand tact et dans le respect de la dignité humaine de tous les peuples.

Après sa mort, la personne perd sa personnalité juridique, elle ne peut plus ester ${ }^{4}$ en justice en son nom. Seuls ses descendants (ou ayants droit) le peuvent. L'appréhension du corps par le droit passe prioritairement par celle du corps vivant. Une fois l'individu mort, le corps se retrouve sacralisé, réifié, il devient une chose, tout en conservant les traces de cette personne juridique. «Si une personne humaine, de son vivant, bénéficie d'un droit à l'inviolabilité et à la protection de son intégrité physique, le postulat de l'intangibilité ne cesse pas pour autant à sa mort $»^{5}$. Ainsi, certains effets juridiques perdurent après la mort de la personne : les articles 225-17 à 225-18-1 du Code pénal sanctionnent les atteintes au respect dû aux morts en prévoyant notamment que l'atteinte à l'intégrité du cadavre soit punie d'emprisonnement ainsi que de $15000 \mathrm{e}$ d'amende. Même si le corps après la mort n'abrite plus juridiquement une personne, des traces de cette personne subsistent et interdisent de traiter le cadavre comme une chose vulgaire (au sens commun du terme).

La Lettre de l'OCIM, 154 | 2016 
Illustration issue du livre de Jérôme Pierrat et Éric Guillon, Le tatouage à Biribi, les vrais, les durs, les tatoués. Clichy : Éditions Larivière, 2004.

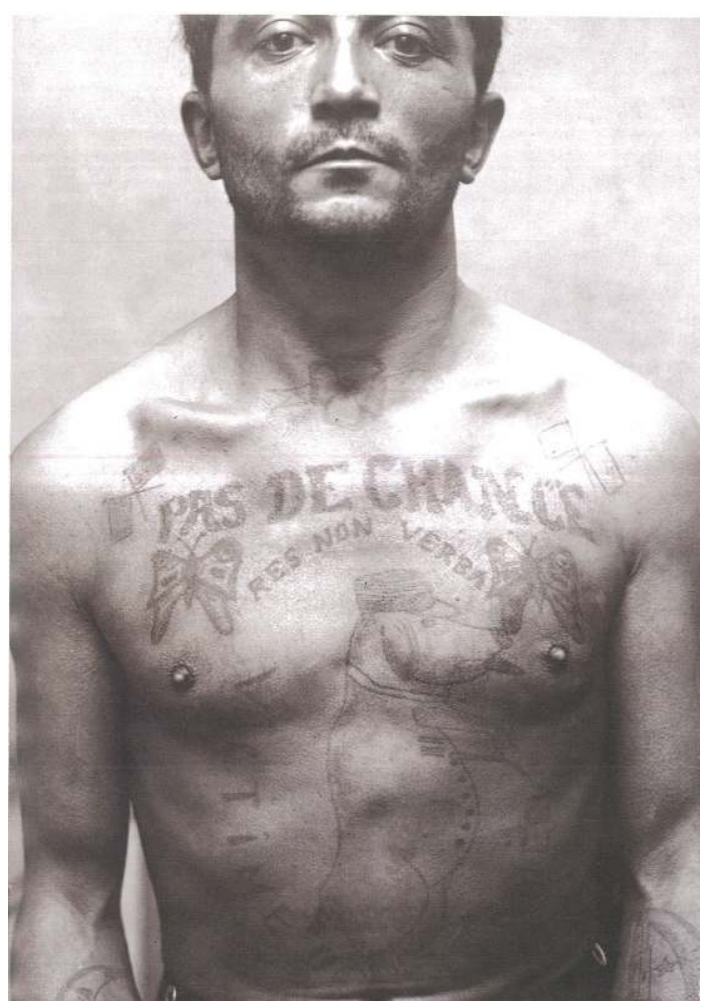

Portrait réalisé entre 1900 et 1930 par les services de l'identité judiciaire et par des médecins criminologues. (C) DR

Selon l'article 16-1 du Code civil, le corps humain est indisponible et non patrimonialisable: "Chacun a droit au respect de son corps. Le corps humain est inviolable. Le corps humain, ses éléments et ses produits ne peuvent faire l'objet d'un droit patrimonial». Pourtant, le corps n'est pas un bien comme un autre: lorsqu'il fait partie du domaine public, les règles d'inaliénabilité, d'incessibilité et d'imprescriptibilité lui sont applicables, comme le prévoit la loi « musée de France », codifiée notamment aux articles L451-1 à L452-4 du Code du patrimoine $e^{6}$, et si le sujet (tout ou partie) est dans ce cas de figure, il peut être assimilé. Le droit reconnaît ainsi qu'un reste humain peut devenir un bien culturel entrant dans le domaine public.

Mais le cadavre n'est pas un objet banal et réclame sa propre disposition, spécialement si l'individu décédé a exposé les conditions de son devenir de son vivant. En effet, il est question du consentement individuel (avant tout usage de son corps ou des parties de son corps par la science) : "Aucun acte médical ni aucun traitement ne peut être pratiqué sans le consentement libre et éclairé de la personne et ce consentement peut être retiré à tout moment " (Code de la santé publique L.1111-4, al.3).

Dans le cas où l'individu décédé n'a pas pu faire part de sa décision concernant un don du corps ou d'organes, c'est la famille ou ses ayants-droits qui vont témoigner de sa volonté. Comme l'a souligné l'ordonnance du tribunal de grande instance de Lille du 5 décembre 1996 :» La dépouille mortelle fait l'objet d'un droit de copropriété familiale inviolable et sacré».

31 Dans le cas où l'individu n'est pas reconnu ou réclamé par sa famille, il est inhumé dans la fosse commune ou dans le carré des indigents ${ }^{7}$. Le corps humain est un objet du droit qui 
interroge les institutions qui le conservent. Dans tous les cas de figure, il est important de savoir que le principe de dignité s'applique toujours.

\section{Reconnaissance patrimoniale}

Le patrimoine est «l'ensemble des biens ou des charges d'héritages qui descendent, suivant les lois, des pères et qui sont légués et transmis aux descendants [...] il est l'ensemble culturel très vaste qui enveloppe les sciences, les langues, les mythes, les institutions humaines [...] mais aussi la totalité des données biologiques qui font l'identité d'une espèce vivante (et spécialement de l'espèce humaine) derrière la variété des différences individuelles $»^{8}$. Le patrimoine est le bien commun d'un État ou d'une Nation, dont les mots d'ordre sont de protéger, conserver, transmettre, étudier, faire connaître, en donnant la possibilité à tout un chacun d'y avoir accès. En France, le patrimoine ne se vend pas, ne se donne pas, mais est conservé pour être transmis aux générations futures.

Dans quelles mesures des corps où fragments de corps deviennent-ils des «objets du patrimoine »?

La première étape au processus de patrimonialisation du corps est la mort, plus précisément, une mort cellulaire, additionnée à une mort sociale. Même dans le cas où l'individu est entouré de sa famille avec un rituel et une cérémonie, on observe que le temps va amener les générations successives à oublier progressivement la disparition de l'individu. Il s'agit de la néantisation. On observe une dissipation des rites mémoriels, tels l'entretien de la tombe ou les visites au cimetière. Le corps perd peu à peu son identité au profit de sa seule dimension matérielle. À partir de ce moment-là, il est seulement lié à une culture, un territoire ou à une structure sociale, et c'est là que se forge le souci patrimonial (même si cela ne signifie pas automatiquement une assimilation en tant qu'objet du patrimoine).

Dans le cas de ces cinq échantillons de peau, le corps n'a pas eu de sépulture, ni de réclamation. Il est ainsi directement passé de l'étape du décès à celle de la néantisation. Alors qu'il n'a jamais été « exploité » lors d'une dissection ou d'une étude postérieure, et qu'il allait sous peu rejoindre au crématorium le reste des spécimens conservés avec lui au congélateur, il s'est retrouvé amputé de sa peau. Aujourd'hui, ce qu'il nous reste c'est cette interface entre lui et nous, ce qui était la preuve de son individualité, mais qui, une fois ôté de son corps, n'est plus tout à fait lui. En ne gardant que la peau, la volonté des préparateurs a été de conserver une part de l'identité du disparu alors même que le processus de néantisation avait déjà œuvré.

Pourtant, même si la volonté des préparateurs a été celle de conserver une part de l'identité du défunt, il n'en demeure pas moins que son identité réelle nous échappe au profit d'une identité suggérée par le biais de l'analyse de ses tatouages 9 . Le statut de ces peaux est ethnographique, elles témoignent d'une époque et de certains usages.

Il en est de ces cinq fragments, comme de toutes les collections de peaux humaines tatouées qui sont conservées à travers le monde : ce ne sont pas seulement des images qui sont conservées, mais ce sont surtout des identités. Ces collections sont cachées, tout comme de nombreuses collections médicinales qui sont aujourd'hui délaissées sous couvert d'une éthique exacerbée, ou de moyens financiers limités.

Pour conclure, même si nous savons que les collections de peaux humaines tatouées sont cachées par les institutions qui les conservent, nous savons aussi qu'elles comportent des 
informations importantes sur notre patrimoine collectif. Ainsi, il est important de prendre en compte leur présence dans les collections qui les conservent. On observe par ailleurs que les mentalités quant à la monstration de ce type d' "objets à caractère patrimonial » tendent peu à peu à évoluer. En témoignent l'exposition en l'an 2000 de ces cinq peaux à la $5^{\mathrm{e}}$ Biennale d'Art contemporain de Lyon (Partage d'exotisme, commissariat de Jean-Hubert Martin), mais aussi les quelques peaux humaines tatouées conservées au musée de l'Homme et exposées au musée du quai Branly (par Anne \& Julien, les fondateurs de la revue Hey) pour l'exposition Tatoueurs, tatoués.

\section{BIBLIOGRAPHIE}

Cadot, L. En chair et en os : le cadavre au musée. Mémoire de recherche de l'École du Louvre, Paris, 2008.

Cartron, I., Castex, D., Georges, P., Vivas, M. et Charageat, M. (dir.) De corps en corps. Traitement et devenir du cadavre. Maison des Sciences de l'Homme d'Aquitaine, 2007-2008.

Caruchet, W. Tatouages et tatoués. Poitiers : Éditions Tchou, 1976.

Delarue J. et Giraud, R. Les tatouages du « milieu ». Paris : Édition La Roulotte, 1950.

Lacassagne, A. Les tatouages : étude anthropologique et médico-légale. Paris : Baillière, 1881.

Lacassagne A. et Magitot, É. Du tatouage, recherches anthropologiques et médico-légales, in Dictionnaire encyclopédique des sciences médicales. Paris : Éditions Masson, Asselin et C $C^{\mathrm{ie}}, 1886$.

Lombroso, C. L'homme criminel. Bocca éditeurs, Libraires de S.M., 2 ${ }^{\mathrm{e}}$ édition, 1898.

Pierrat, J. et Guillon, É. Le tatouage à Biribi, Les vrais, les durs, les tatoués. Clichy : Éditions Larivière, 2004.

\section{NOTES}

1. Locard, E. Traité de criminalistique. Tome 3 Les preuves de l'identité, Chapitre 5 «Le tatouage», Lyon, 1932, pp. 249-428.

2. Lacassagne, A. et Magitot, É. «Tatouage», in Dechambre, A. Dictionnaire encyclopédique des sciences médicales. $3^{\mathrm{e}}$ série, tome XVI, Paris : Éditions Masson, Asselin et C $C^{\mathrm{ie}}, 1886$.

3. Hormis les deux peaux des dessus de pieds qui ont été positionnées sur la même planche.

4. Ester : agir en justice.

5. Coulet, J. et Lacroix, M. De l'»être «vers l'»avoir été»: Muabilité du corps humain au moment du décès, Lex Electronica, vol. 15, n², automne 2010.

6. Le service public de la diffusion du droit, Code du patrimoine. Légifrance : www.legifrance.gouv.fr

7. Le carré des indigents est une parcelle réservée dans certains cimetières aux défunts dont les corps ne sont pas réclamés par les proches. Il abrite des sépultures sans nom et sans pierre tombale. Une simple dalle de béton ferme chaque fosse. 
8. Frangne, P.-H. Patrimoine, sources et paradoxes de l'identité, in Actes du cycle de conférences prononcées à l'université Rennes 2, 5 novembre 2007-2 avril 2008, Andrieux, J.-Y. (dir.) collection «Art et société», Presses universitaires de Rennes, 2011, p. 266.

9. Quétel, É. De la conservation d'un patrimoine caché : cinq peaux humaines tatouées, de l'anthropologie criminelle à l'intention esthétique. Mémoire de fin d'études à l'ESAA, 2013.

\section{RÉSUMÉS}

Cet exemple d'une étude de conservation-restauration de cinq peaux humaines tatouées, conservées au musée d'Anatomie Testut-Latarjet de Lyon, permet d'aborder la problématique de la conservation et de l'exposition des restes humains autour de deux grandes questions : leur statut juridique et leur reconnaissance patrimoniale.

\section{AUTEUR}

\section{PAR ÉLOÏSE QUÉTEL}

Éloïse Quétel est conservatrice-restauratrice d'objets ethnographiques et de restes humains queteleloise@yahoo.fr 